\title{
Germanica
}

\section{Le destin romanesque des Juifs allemands dans l'œuvre de Jakob Wassermann : entre quête d'identité et isolement}

Das romanhafte Schicksal der deutschen Juden zwischen Identitätssuche und Isolierung

Martine-Sophie Benoit

\section{(2) OpenEdition}

\section{Journals}

Édition électronique

URL : http://journals.openedition.org/germanica/1323

DOI : 10.4000/germanica.1323

ISSN : 2107-0784

Éditeur

Université de Lille

Édition imprimée

Date de publication : 30 juin 1998

Pagination : 93-105

ISBN : $9770984263203-22$

ISSN : 0984-2632

Référence électronique

Martine-Sophie Benoit, « Le destin romanesque des Juifs allemands dans l'œuvre de Jakob Wassermann : entre quête d'identité et isolement », Germanica [En ligne], 22 | 1998, mis en ligne le 31 janvier 2012, consulté le 06 octobre 2020. URL : http://journals.openedition.org/germanica/1323 DOI : https://doi.org/10.4000/germanica.1323

Ce document a été généré automatiquement le 6 octobre 2020.

(c) Tous droits réservés 


\title{
Le destin romanesque des Juifs allemands dans l'œuvre de Jakob Wassermann : entre quête d'identité et isolement
}

\author{
Das romanhafte Schicksal der deutschen Juden zwischen Identitätssuche und \\ Isolierung
}

Martine-Sophie Benoit

1 Jakob Wassermann aurait voulu délimiter sa production romanesque par deux ouvrages : Die Juden von Zirndorf aurait été le premier maillon de cette chaîne (l'auteur interdit de son vivant la réimpression de tout écrit antérieur) ${ }^{1}$; le dernier maillon devait quant à lui développer «l'histoire de la vie de Ahasvérus", illustrant ainsi « le destin juif " à la lumière de discussions entre le Juif errant et des personnages de l'histoire convoqués pour la circonstance: St Paul, Spinoza, Napoléon, Karl Marx, Lénine, Hitler². Même si Die Juden von Zirndorf n'est pas le premier roman de Wassermann et que Die Lebensgeschichte des Ahasver est demeuré à l'état d'esquisse, on voit sous quel signe Jakob Wassermann aurait aimé s'inscrire dans l'histoire littéraire allemande : celui du dialogue mais aussi de la confrontation entre Juifs et non-Juifs.

2 Né dans la communauté juive de Furth en Bavière, de mère et de père juifs, Jakob Wassermann se situe d'emblée dans une situation délicate à assumer : refusant d'être renégat, il dénonça toute sa vie les compromis de l'assimilation qui tendent à une négation de soi et de l'identité juive; mais ancré dans la culture allemande, il ne se reconnaît pas dans le mouvement sioniste et son projet de renaissance nationale ${ }^{3}$. La revendication sioniste lui paraît d'ailleurs si étrangère qu'aucun de ses personnages romanesques ne s'en fait l'écho. En revanche, désireux de construire une œuvre romanesque contemporaine à la manière d'un Balzac, Jakob Wassermann témoigne dans de nombreux romans d'actualité ${ }^{4}$ de la difficile réalité vécue par les Juifs en Allemagne dans le premier tiers $\mathrm{du} \mathrm{xx}^{\mathrm{e}}$ siècle. 
3 Il nous montre ainsi des enfants, des femmes et des hommes confrontés au conflit intérieur qui affectait alors tout Juif allemand: d'un côté, les institutions juives perpétuaient la tradition d'une minorité persécutée; de l'autre, la société allemande manifestait toujours plus d'hostilité à l'intégration et à l'assimilation.

4 Je me propose d'analyser ici cet insoluble dilemme à travers les différents personnages juifs présents dans l'œuvre de JakobWassermann - c'est-à-dire de montrer comment l'auteur présente les relations entre individus (entre Juifs, et entre Juifs et non-Juifs), quel chemin il fait emprunter à ses personnages, si c'est celui du reniement, du repli sur soi, de l'abandon de soi ou de l'isolement. Pour ce faire, j'étudierai tout d'abord l'environnement dans lequel évoluent ces figures juives, pour tenter ensuite de mieux cerner leurs contours et leurs caractéristiques. L'objet de l'article est de mettre en évidence le regard que Jakob Wassermann, Juif qui, comme l'écrivait Kurt Tucholsky, était "plus allemand que les Allemands eux-mêmes", porte sur cette minorité allemande peu de temps avant son isolement juridique et son extermination.

\section{Face à l'hostilité de la population}

Dans les romans de Jakob Wassermann, les rapports des personnages juifs avec leur environnement allemand non-juif laissent une large place à l'attitude d'hostilité antisémite. L'antisémitisme n'est pas le seul fait de personnages identifiés mais est décrit comme un phénomène répandu dans la population et jusque dans les institutions du pays : Jakob Wassermann met en œuvre de nombreuses situations romanesques où l'un des personnages juifs est poursuivi par un groupe d'individus aux fameux cris antisémites de "Hepp, Hepp " (voir Christian Wahnschaffe, 441,624; Joseph Kerkhovens dritte Existenz, 57) ; l'auteur évoque la montée de l'antisémitisme dans le milieu scolaire (cf.: Der Fall Maurizius, 309), universitaire (Das Gänsemännchen, 65 ; Der Fall Maurizius, 307-309; EtzelAndergast, 451-452) et parlementaire: lors d'une interpellation du gouvernement sur l'affaire Jutta Elasser, jeune Juive enlevée et enfermée dans un couvent, le public ainsi que de nombreux députés bloquent le débat en déclenchant un brouhaha général (Der Moloch, 94). Par la diversité de ses personnages antisémites, Jakob Wassermann témoigne de l'ampleur de cette haine dans toutes les couches de la population allemande. L'antisémitisme des petites gens, la plupart du temps basé sur l'antijudaïsme chrétien, apparait sous des traits souvent vulgaires et répulsifs : Sürich Sperling, l'aubergiste de Zirndorf, concentre en sa personne tous les vices et les tares de l'antisémite ignare, grossier et cruel (on notera qu'il est le premier personnage de ce type à apparaître sous la plume de Wassermann); le boucher Vravar, personnage du roman Der Moloch, réussit à monter les villageois de Podolin contre Samuel Elasser, malheureux colporteur juif, père de la jeune Jutta; le petit-bourgeois Carovius, personnage peu amène de Das Gänsemännchen, se plonge dans la lecture des ouvrages du scientifique Friedrich Benda afin de mieux combattre ce Juif « qui ne ressemble pas à un Juif, plutôt à un Hollandais " (Das Gänsemännchen, 64); Amadeus Voss, fils d'un garde-champêtrequi éloigne le jeune aristocrate Christian Wahnschaffe de sa famille et de ses amis, est un catholique fanatique qui dit haïr la " race juive ", étrangère selon lui au peuple allemand, et lie cette haine du Juif à ses croyances chrétiennes (Christian Wahnschaffe, 457) ; enfin Niels Heinrich Engelschall, assassin odieux de la petite Ruth Hofmann, méprise cette jeune fille "étrangère, de race étrangère, de sang [...] étranger » (Christian Wahnschaffe, 413). 
6 Mais l'hostilité antisémite n'est pas uniquement le lot de personnages caricaturaux et repoussants, Jakob Wassermann thématise aussi l'antisémitisme « de bon ton » d'une part de la bourgeoisie et de l'aristocratie allemandes. Le journaliste Stefan Gudstikker et l'enseignant Bojesen, tous deux pourtant proches d'Agathon Geyer, le personnage central du roman Die Juden von Zirndorf, se répandent en invectives contre l'influence délétère des Juifs sur l'Allemagne et sa culture (Die Juden von Zirndorf, 106, 123). L'antisémitisme aristocratique est mis en relief dans Der Moloch: lors d'un repas mondain, le jeune héros Arnold Ansorge se voit attaqué pour son soutien au colporteur Samuel Elasser car, explique son détracteur, « il faut bien un peu fouetter cette racaille juive pourrie : ces gens n'ont pas accès à notre sensibilité si subtile, la jeune enfant sera mille fois mieux dans le couvent que dans l'étable dans laquelle elle a grandi » (Der Moloch, 395). La mère de Christian Wahnschaffe réprouve quant à elle tout lien avec des Juifs et ses amis ne comprennent d'ailleurs pas pourquoi le jeune aristocrate se préoccupe du meurtre d'une Juive (Christian Wahnschaffe,88, 533).

7 Cette sombre peinture d'une société allemande imprégnée par l'antisémitisme se trouve toutefois pondérée par la manifestation d'une sympathie philosémite.

\section{Le soutien philosémite}

8 C'est aux personnages centraux des romans de JakobWassermann qu'il revient de donner la réplique à cette haine ambiante. La jeune Allemande Renate Fuchs donne naissance à Beatus, fils d'Agathon Geyer(Die Geschichte der jungen Renate Fuchs) ; Arnold Ansorge part à Vienne pour qu'on rende justice au colporteur Samuel Elasser (Der Moloch) ;Sylvester von Erfft, «l'homme de quarante ans » exempt de tout préjugé, tombe amoureux d'une jeune Juive (Der Mann von vierzig Jahren); Daniel Nothafft est l'ami intime du scientifique Friedrich Benda qu'il considère un peu comme un frère (Das Gänsemännchen); ce même Nothafft reçoit des lettres d'une jeune Juive, Regine Sussman, qu'il juge « extraordinaire » (Das Gänsemännchen, 414); Christian Wahnschaffe réussit à faire la lumière sur l'horrible assassinat de la jeune Ruth Hofmann (Christian Wahnschaffe); Etzel Andergast a, malgré les préventions de son père, des camarades juifs à l'école (Der Fall Maurizius)et se lie ultérieurement d'amitié avec Max Mewer, jeune Juif à la destinée tragique (Etzel Andergast). En quelque sorte porte-parole de tous ces personnages, Etzel Andergast se demande «quel satan a bien pu piquer (les antisémites) ? Ils haïssent, et pourquoi ? Parce qu'ils haïssent ! (Etzel Andergast,454).

9 Le profil de ces personnages fait cependant question car ce sont des hommes et des femmes qui vivent en marge de leur groupe social ou familial : Renate Fuchs et Arnold Ansorge refusent les contraintes de la vie bourgeoise hypocrite; Sylvester von Erfft, Christian Wahnschaffe et Etzel Andergast sont tous trois en rupture de ban avec leur milieu aristocratique sclérosé ; Daniel Nothafftincarne l'artiste mal aimé, rejeté, qui attire sur lui l'hostilité de son environnement. On voit alors où le bât blesse : comment le Juif peut-il s'intégrer dans la société allemande si seuls des marginaux sont la caution de cette intégration? Friedrich Benda, scientifique déçu, relate avec amertume la situation dans laquelle sont confinés les Juifs allemands :

Le moins qu'(un Juif) ait à craindre, c'est une distance lourde de préjugés, comme si

l'autre lui criait: moi je suis ici, toi tu es là-bas - n'emprunte pas le pont. (Das

Gänsemännchen, 71). 


\section{Caractéristiques des personnages juifs}

10 Pour comprendre comment les personnages juifs réagissent à leur environnement allemand, il faut désormais entrer plus avant dans leur description. Est-ce volonté objective de la part de JakobWassermann de décrire les préjugés de l'intérieur ou, de manière plus ambiguë, de s'associer à un rejet d'une tradition et d'une culture juives jugées obsolètes, toujours est-il que deux traits significatifs caractérisent la présentation des Juifs chez cet auteur: d'une part, ils sont identifiables par leur apparence physique; d'autre part, ce sont des individus isolés, coupés de leur communauté d'origine.

11 Dans Das Gänsemännchen, Carovius, un des personnages identifié comme antisémite, explique que l'ami juif de Daniel Nothafft, Friedrich Benda, ne ressemble nullement à un Juif: Benda «n'est certes pas tout à fait blond mais n'a pas non plus les cheveux noirs » et «son nez est aussi droit qu'une règle » (Das Gänsemännchen, 64). À l'inverse, Waremme, personnage juif étrange et déroutant dont le faux témoignage a valu à Maurizius l'emprisonnement à perpétuité, explique que c'est justement son apparence physique qui lui a permis de jouer un rôle dans la société : « La nature m'avait avantagé, j'étais blond, d'un blond authentiquement germanique, la forme de mon visage [...] n'est pas orientale » (Der Fall Maurizius, 311). Ces deux citations sont révélatrices d'un détail qui peut paraître aujourd'hui troublant mais qui, au début du siècle, passait pour banal: les personnages juifs sont pour la plupart physiquement identifiés - par la couleur de leurs cheveux ou la forme de leur nez, pour les femmes aussi par la pâleur de leur visage : Miriam Geyer, sœur d'Agathon (Die Geschichte der jungen Renate Fuchs, 392-393), et Olga Veit, amoureuse malheureuse d'un dirigeant communiste (Faber, 212), ont les cheveux noirs et le teint aussi pâle que Ruth Hofmann (Christian Wahnschaffe, 398); Wurzelmann et Regina Sussmann, personnages secondaires du roman Das Gänsemännchen, apparaissent tous deux comme le type même du "Juif rouquin " (Das Gänsemännchen, 94,414); Edward Nieberding, prototype du Juif assimilé qui renie ses origines, a des "lèvres charnues" et un "gros nez" (Die Judenvon Zirndorf, 149); Johanna Schöntag, une amie de Christian Wahnschaffe, est caractérisée par sa laideur et notamment la « hideur» de son nez (Christian Wahnschaffe, 243-244); Gisa Schumann, une des femmes juives gravitant autour de Renate Fuchs, a « un visage passionné aux lignes orientales » (Die Geschichte der jungen Renate Fuchs, 25); enfin dans une lettre à sa femme, Sylvester von Erfft, "l'homme de quarante ans ", décrit la jeune Rachel, «l'Orientale langoureuse » dont « les yeux noirs de Juive (lui) content la douleur de tant de générations » (Der Mann von vierzig Jahren, 19-20). On voit ainsi se détacher la silhouette des personnages juifs reconnaissables à leur seule apparence qui serait «typiquement » juive. Un ami de Etzel Andergast, Max Mewer, «homme laid de type juif prononcé » (Etzel Andergast, 410) parle de ses particularités physiques comme d'une « incurie ethnique » (Etzel Andergast, 451). On pourrait varier cette expression et parler chez Jakob Wassermann d'une « apparence ethnique » caractérisant bon nombre de ses figures juives.

12 À cette distinction physique qui ne manque pas aujourd'hui de mettre quelque peu mal à l'aise, vient s'ajouter une caractéristique, inquiétante elle aussi: Agathon Geyer, personnage central du roman Die Juden von Zirndorf, est le seul personnage juif que Jakob Wassermann laisse évoluer dans un milieu juif. On voit vivre Agathon au sein du groupe familial, dans ses contacts avec d'autres enfants et adultes juifs. Or ce qui 
caractérise le jeune homme, c'est la révolte contre sa communauté - une révolte tant culturelle que religieuse, qui le pousse à s'émanciper de toute tutelle. Agathon a tout d'abord perdu tout lien religieux. Il méprise ces hommes dont les visages à la sortie de la synagogue, loin de refléter un quelconque recueillement intérieur, respirent «l'ardeur du marchandage et la foi hypocrite » (Die Juden von Zirndorf, 86). Pour lui, la religion juive est "vieille", c'est "quelque chose de mort, d'impossible à ressusciter, quelque chose de figé, de spectral " (Die Juden von Zirndorf, 86). La rupture est aussi culturelle: Agathon reproche aux membres de la communauté leur manque de respect de soi, leur attitude obséquieuse :

Vous autres, vous acceptez vraiment tout. Ils peuvent vous arracher les oreilles, vous leur baisez la main. Il n'y a guère qu'à la maison que vous sachiez pester ${ }^{5}$. (Die Juden von Zirndorf, 70)

13 L'histoire d'Agathon Geyer, premier personnage juif central de l'œuvre romanesque de Jakob Wassermann, apparaît en quelque sorte comme la prémisse de la vie de toutes les autres figures juives. Présentés en dehors de tout contexte de vie juive, les personnages juifs ne sont soudés par aucune appartenance religieuse ou culturelle, ce sont des êtres déchirés et isolés - des sortes de "Luftmensch». La question est alors de savoir comment ces êtres réagissent à cet isolement, si cet isolement les pousse vers un repli sur soi ou si les tentatives d'intégration dans le milieu allemand sont réitérées. On s'aperçoit alors que Jakob Wassermann traite de manière fort différente les personnages de femmes et d'enfants d'une part, d'hommes d'autre part. C'est de cette différenciation qu'il nous faut traiter maintenant, tout en nous interrogeant sur sa finalité et sur le message que Wassermann entend nous transmettre.

\section{Des enfants et des femmes au destin tragique}

Si on tente de caractériser de manière générale les femmes et les enfants juifs dans l'œuvre de Wassermann, on peut dire que ceux-ci apparaissent comme des figures élégiaques dont la beauté et la grandeur d'âme sont assombries par un destin tragique.

Les enfants sont les premiers touchés par le destin. La petite Jutta Elasser dont « le regard mélancolique » attire l'attention d'Arnold Ansorge (Der Moloch, 31) est arrachée à sa famille et, bien qu'elle réussisse à faire parvenir à celle-ci une missive, " un cri de détresse » (ibid., 125), elle reste prisonnière des religieuses décidées à faire d'elle une chrétienne. Le petit Chaim Papier, recueilli par Marie, la femme de Joseph Kerhoven, tombe malade à l'idée d'être maltraité par des gamins antisémites (Joseph Kerhovens dritte Existenz, 57-61). Miriam Geyer, «jeune fille sans repos ni bonheur » (Die Geschichte der jungen Renate Fuchs, 520), reprend, à la mort de son frère Agathon, une vie faite d'errances et d'insécurité. Le jeune Max Mewer, ami de Etzel Andergast, et le petit Michael Hofmann, jeune Juif craintif miné par les sentiments de haine à son égard, doivent faire face à la mort du frère pour le premier, de la sœur pour le second: Hermann Mewer s'est suicidé le jour où la corporation estudiantine qu'il avait luimême fondée l'exclut (Etzel Andergast, 451-452); Ruth Hofmann, qui trouvait « la vie si merveilleuse, si profondément merveilleuse, si riche et si puissante " (Christian Wahnschaffe, 459) et donnait tout son temps aux souffreteux et aux laissés-pour-compte de la société, est sauvagement assassinée par un ouvrier.

Les personnages féminins sont eux aussi douloureusement touchés par le destin. Certaines connaissent des amours malheureux qui les détruisent: Regina Sussmann, 
qui reconnaît en Daniel Nothafft le génie que le monde se refuse de découvrir en lui, cette femme «nerveuse, douce» et "merveilleuse» (Das Gänsemännchen, 414) se consume dans cet amour à sens unique; Gisa Schumann, amie de Renate Fuchs, est décrite comme " un miracle de tendresse et de noblesse intérieure ", une «capitaliste du cœur " (Die Geschichte der Renate Fuchs, 28) mais vit un amour malheureux avec un officier; la belle Rachel, "Orientale langoureuse " à "l'enthousiasme rêveur ", ne fascine que pour un temps Sylvester von Erfft et sombre dans la folie (Der Mann von vierzig Jahren, 20, 30); Olga Veit enfin est poussée au suicide par un dirigeant communiste dont elle attend un enfant (Faber, 236-237). Un peu à part, Johanna Schöntag semble cristalliser en elle tout le tragique de ces destinées : amante passagère de Christian Wahnschaffe, elle est une personne très attentive aux autres, «rien n'échappe à son regard attentif» (Christian Wahnschaffe, 256 - elle aide notamment Michael Hofmann à surmonter l'assassinat de sa sœur Ruth); mais incapable de s'accepter elle-même, elle est dévorée par une « haine de soi » (590) destructrice, par « un mépris suicidaire » (507) et aspire à la mort : « Mourir, pensait-elle, mourir, c'est la seule chose qui reste » (624).

Alors que les femmes et les enfants sont décrits sous des traits tragiques et font figures de victimes, les hommes tentent de leur côté d'agir ou de réagir.

\section{Des hommes qui forcent le destin}

Les caractères masculins jouent un rôle actif dans l'œuvre romanesque de Jakob Wassermann, au risque de commettre des erreurs et des actes regrettables. Aussi certains personnages masculins apparaissent sous un jour défavorable: ainsi Dingolfinger, mauvais médecin incapable de sauver Lenore, la seconde femme de Daniel Nothafft, et son enfant lors de l'accouchement (Das Gänsemännchen, 360-361) ; ou bien le Juif Menasse qui, dans Der unbekannte Gast, tente en vain de servir de médiateur entre les aristocrates et les révolutionnaires lors de la révolution russe ; Edward Nieberding aussi qui, «Juif moderne qui a cessé d'être juif », inspire le mépris(Die Juden von Zirndorf, 179); ou bien Wurzelmann,Juif de l'Est aux cheveux roux et parlant yiddish, sorte d'imprésario malhonnête qui à la fois soutient et accable Daniel Nothafft (Das Gänsemännchen, 162, 245) : c'est un homme envieux (il se définit lui-même comme «le génie de la jalousie », 163) et obséquieux - d'aucuns l'appellent « le larbin » (162).

Abstraction faite de ces personnages somme toute très marginaux dans la trame des romans, on peut faire la distinction entre des personnages renvoyant à des figures paternelles d'une part et les jeunes hommes et hommes d'âge mûr d'autre part.

Le vieux Gedalja est, dans Die Juden von Zirndorf, une véritable figure de patriarche: renié par son fils qui, devenu baron, dissimule aux yeux de la société ce père sorti tout droit du ghetto et incapable de parler un allemand correct, Gedalja sert de repère au héros du roman, Agathon Geyer. Gedalja est le seul personnage du roman à transmettre au jeune homme une vision positive de la croyance en un Dieu suprême : selon lui, contrairement à l'enseignement à la synagogue, la Bible "doit être bue comme du vin ", « elle ne doit pas être lue avec les yeux du corps mais avec ceux de l'esprit » (Die Juden von Zirndorf, 117). On pourrait rapprocher de Gedalja le pauvre colporteur Samuel Elasser. Quoique très obséquieux, Elasser ne s'en laisse pas compter, il ne craint par le boucher antisémite Vravar auquel il réclame de l'argent et tente toutes les démarches pour faire libérer sa fille: il intente un procès, demande une audience auprès du 
ministre de la justice et même de l'Empereur. Mais bien qu'admirables de courage et de grandeur, Gedalja et Elasser ne proposent pas de projection dans l'avenir: Gedalja appartient trop entièrement au passé, avec lui mourra le dernier reliquat de religion dans la communauté juive de Zirndorf; quant à Elasser, il ne réussit pas à sauver sa petite fille Jutta.

21 À ces pères respectueux de l'autorité répondent trois figures d'hommes plus ou moins jeunes qui ont en commun de franchir le pont qui sépare le Juif du non-Juif : il s'agit d'Agathon Geyer, le héros de Die Juden von Zirndorf, de Friedrich Benda, l'ami de Daniel Nothafft, et de Waremme/Warschauer, témoin à charge dans le procès Maurizius.

Waremme/Warschauer, né en Silésie, choisit d'abord la solution de la conversion, l'abandon de toute référence juive : enfant, il s'invente une mère chrétienne, prétend être le fruit d'un adultère avec un grand propriétaire noble de Silésie; de son père adoptif, catholique fervent, il prend le patronyme de Waremme qui lui ouvre les portes du monde, jusqu'au pape et à l'Empereur. Mais ses propres illusions s'effondrent sous le coup d'une déception amoureuse. Waremme part alors à la recherche de Warschauer et se jette sur la route : à Chicago il noue des contacts avec des Juifs hassidiques, se plonge dans les études bibliques puis reprend le chemin en sens inverse. Il retourne à Berlin (c'est là qu'a lieu la rencontre avec le jeune Etzel Andergast qui éclaircit l'« Affaire Maurizius ») d'où il veut continuer « vers l'Est, vers l'Est » (Der Fall Maurizius,495) : en Haute-Silésie polonaise vit une de ses filles, peut-être pourra-t-il trouver là « une sorte de lieu de repos" (495). À travers Warschauer semble ainsi dressé le constat de l'impossible "symbiose judéo-allemande », auquel répond la tentative d'assumer ses origines en partant à la recherche des racines perdues.

Entravé dans sa carrière universitaire du seul fait de ses origines, Friedrich Benda a quant à lui fui l'antisémitisme et gagné l'Afrique pour poursuivre des recherches scientifiques qui le passionnent. Après dix-sept années, il rentre dans sa ville natale et déclare vouloir y rester définitivement, malgré l'antisémitisme toujours virulent :

Tout avance très, très lentement. L'humanité est encore une enfant et nous exigeons d'elle la justice. Justice! Quel chemin reste-t-il encore à parcourir pour y arriver! Aussi long que de la forêt vierge au jardin. Nous devons avoir de la patience pour les nombreuses générations qui nous suivront (Das Gänsemännchen, 484).

Par cette affirmation, Benda manifeste sa foi en la tolérance et la raison; il est cependant le seul de ces trois personnages à retourner en Allemagne pour y mourir.

L'histoire d'Agathon Geyer se veut la répétition réussie de la vie du faux prophète Sabbatai Sewi : alors que ce dernier a trahi son peuple en se convertissant finalement à l'Islam, Agathon devient une figure rédemptrice : il assassine l'aubergiste antisémite, geste symbolique devant libérer la terre du sentiment de haine contre les Juifs; dès lors, il est « rédempteur » (Die Juden von Zirndorf, 156), une sorte de « jeune Jésus » (ibid., 154) qui prêche l'amour du prochain et s'oppose avec succès à la haine antisémite (ibid., 162); il rappelle sa mère à la vie (ibid., 144) et redonne espoir à la malheureuse Renate Fuchs (Die Geschichte der jungen Renate Fuchs). Après un séjour en Galicie où il apporte de l'aide à ses coreligionnaires en détresse (Die Geschichte der jungen Renate Fuchs, 475), Agathon, alors en Moravie, tombe gravement malade : il a « un cœur épuisé, un cœur trop usé » pour survivre. Avant de mourir dans les bras de Renate Fuchs, il s'unit à elle. De cette union naît Beatus, enfant de l'espoir en un rapprochement judéo-allemand radieux. 


\section{Conclusion}

Le tableau que les romans de Jakob Wassermann dressent du Juif en Allemagne dans le premier tiers $\mathrm{du} \mathrm{xx}^{\mathrm{e}}$ siècle sensibilise le lecteur par son aspect à la fois lumineux et funeste: les Juifs apparaissent pour la plupart comme des personnages qui forcent l'admiration par leur grandeur d'âme, leur propension à l'entraide - mais leur chemin mène le plus souvent à la mort, réelle ou symbolique. Coupés de leurs racines familiales, religieuses et ethniques, ces hommes, ces femmes et ces enfants sont isolés et dans l'impossibilité d'affirmer une autonomie que la population chrétienne leur refuse largement. Si les personnages féminins et enfantins sont trop marqués par le destin pour porter en eux un message d'espoir, Jakob Wassermann nous livre au travers de trois figures masculines - Agathon Geyer, Benda et Warschauer - des perspectives qu'il semble envisager afin de rapprocher Juifs et non-Juifs. La chronologie quant à elle révèle l'évolution de l'auteur à ce sujet : l'union d'un Juif allemand, Agathon, et d'une chrétienne allemande, concrétisée par la naissance de Beatus, est racontée au début du siècle ; l'espoir en une tolérance possible et une cohabitation sereine, symbolisée par le retour de Benda en Allemagne, est évoqué dans un roman écrit avant la Première Guerre mondiale ; l'impossibilité d'un rapprochement judéo-allemand et la recherche d'une identité "orientale», incarnée par Warschauer, sont développées en 1928 comme si, lentement mais inexorablement, montait en Jakob Wassermann le doute d'une quelconque réussite de cette "symbiose judéo-allemande » tant désirée mais si peu concrétisée. La solution romanesque s'impose alors: puisque le Juif ne cessera jamais d'être juif pour les autres, puisque l'isolement et le repli sur soi ne peuvent être des solutions, il revient au Juif lui-même d'affirmer sa judéité et de partir à la recherche de sa propre identité.

\section{NOTES}

1. Jakob Wassermann, Die Juden von Zirndorf, 1ère édition en 1897 chez Albert Langen - réédition en poche en 1996 chez DTV (les références renvoient à cette réédition).

2. Lettre de Jakob Wassermann datée du 25.8.1933, citée dans : Dierk Rodewald, Jakob Wassermann (1873-1934) - Ein Weg als Deutscher und Jude -Lesebuch zu einer Ausstellung, Bouvier (Bonn, 1984), p. 142.

3. En ce qui concerne la position de Wassermann sur le sionisme, on pourra se référer à son texte autobiographique Mein Weg als Deutscher und Jude paru en 1921 chez Fischer (voir notamment les pages 105-108 dans la réédition chez DTV). Cette attitude de refus était affichée par Wassermann dès le début du siècle(voir une lettre à Georg Brandes du 21.12.1901 : «Je ne suis absolument pas sioniste, je suis personnellement et intellectuellement tout à fait insensible à ces agissements insensés ", Dierk Rodewald, op. cit., p. 41)

4. Après lecture de toute l'œuvre romanesque de Jakob Wassermann, neuf « Zeitromane » (tous parus chez Fischer) retiennent l'attention pour l'étude des personnages de Juifs chez cet auteur ; il s'agit de : Die Juden von Zirndorf (cf. : note 1); Die Geschichte der jungen Renate Fuchs (1900); Der 
Moloch (1903) ; Der Mann von vierzig Jahren (1913) ; Das Gänsemännchen (1915-réédition DTV 1990) ; Christian Wahnschaffe (1919-réédition DTV 1989) ; Faber oder die verlorenen Jahre - Der Wendekreis, 4. Folge (1924); Der Fall Maurizius (1928-réédition DTV 1988) ; Etzel Andergast (1931-réédition DTV 1988) ; Joseph Kerkhovens dritte Existenz (1934-réédition DTV 1989). J'ai exclu Engelhart oder die zwei Welten, texte commencé par Wassermann dès 1902 et édité de manière posthume en 1973 (Langen Müller, München) car il s'agit plus d'une autobiographie romancée que d'un roman. Les références de pages données dans le texte renvoient, le cas échéant, aux rééditions.

5. Furieux de ne pouvoir se reconnaitre dans ce peuple pour lequel il ressent à la fois de l'affinité et de la haine (cf. 201), Agathon commettra le geste irréparable, qui le coupe de tout lien, en assassinant l'aubergiste antisémite Sürich Sperling.

\section{RÉSUMÉS}

Cet article se donne pour objet l'étude de la crise existentielle vécue par la minorité juive en Allemagne dans le premier tiers de $\mathrm{XX}^{\mathrm{e}}$ siècle telle qu'elle a été interprétée par Jakob Wassermann. Né dans la communauté de Furth en Bavière, de mère et de père juifs, Jakob Wassermann s'est d'emblée placé dans une situation délicate, refusant tout à la fois l'assimilation - vécue comme une négation des origines - et le sionisme - qu'il ne comprend pas. Je voudrais montrer comment à travers neuf romans d'actualité, Jakob Wassermann tente d'analyser les conflits intérieurs des Juifs allemands face à leur situation, leur tentative d'échapper à l'isolement auquel semble les condamner une société allemande hostile.

In diesem Artikel möchte ich die Lebenskrise der jüdischen Minderheit in Deutschland im ersten Drittel des XX. Jahrhunderts studieren, so wie sie von Jakob Wassermann in seinen Romanen dargestellt wurde. In der jüdischen Gemeinde von Fürth in Bayern geboren, befand sich Jakob Wassermann von Anfang an in einem schwierigen Zwiespalt: er lehnte gleichzeitig die Assimilation - die er als Negierung seiner Herkunft empfand - und den Zionismus ab - der ihm fremd blieb. Ich möchte also zeigen, wie der Schriftsteller in neun seiner Zeitromane die inneren Konflikte der deutschen Juden beschreibt, ihren Versuch, der Isolierung zu entrinnen, zu der sie eine feindlich gesinnte deutsche Umgebung zu verdammen schien.

\section{AUTEUR}

\section{MARTINE-SOPHIE BENOIT}

Université Charles-de-Gaulle - Lille 3 